\title{
The Use of Chemical Design Tools to Transform Proteomics Data into Drug Candidates
}

\author{
Philip M. Dean and Edward D. Zanders \\ De Novo Pharmaceuticals Ltd., Cambridge, UK
}

Computational Proteomics Supplement 32:S28-S33 (March 2002)

\begin{abstract}
The Human Genome Project has fueled the massive information-driven growth of genomics and proteomics and promises to deliver new insights into biology and medicine. Since proteins represent the majority of drug targets, these molecules are the focus of activity in pharmaceutical and biotechnology organizations. In this article, we describe the processes by which computational drug design may be used to exploit protein structural information to create virtual small molecules that may become novel medicines. Experimental protein structure determination, site exploration, and virtual screening provide a foundation for small molecule generation in silico, thus creating the bridge between proteomics and drug discovery.
\end{abstract}

\section{INTRODUCTION}

It is now less than one year since the landmark papers describing the sequencing of the human genome $(6,13)$, and we are now firmly in the new post-genomic world facing up to the following problem: how do we maximize the use of the newly acquired data for improving healthcare? It has long been realized that genomic sequence alone will not hold the key, but that a systematic high-throughput analysis of protein expression, interactions, and structure (proteomics) is required. The scale of the problem is illustrated by the fact that the number of therapeutic targets available for drug discovery will increase from the current number of 600-1000 to perhaps as many as 500010000 , thus putting huge demands on already inefficient discovery processes.

In order to meet these challenges, the information gained from protein structure determination needs to be used to create new molecules in silico on a scale that is impossible to contem plate using synthesized molecules and high-throughput screening. New computer algorithms have been written to approach the complex task of building small molecules de novo within a ligand-binding site; these take account of all interactions between the ligand and the site. Where the protein structure is not available, a virtual binding site may be built in silico using binding data from existing ligands and used to design molecules in the same fashion. These processes of structure-based design and ligand-based design (SBD and LBD, respectively) are described, from input of protein data to output of small molecule structures.

\section{STRATEGIES FOR DRUG DESIGN}

Figure 1 illustrates the connections between protein targets and drug design.

\section{Validated Protein Targets}

A detailed discussion of target validation is outside the scope of this article but has been reviewed in many publications (e.g., 4). If the expression of a protein is altered in disease, or an animal model of pathology, then it may be considered a potential target for drug design. On the other hand, if the pathological role of a protein is unknown, then a small molecule inhibitor could be a useful functional probe; this area has been recently named chemical genomics.

\section{Protein Structure Determination}

X-ray crystallography remains the primary method for generating a three-dimensional (3-D) protein structure, but the technical issues remain formidable. Nevertheless, there have been improvements in protein production and purification, crystallization, X-ray analysis, and data reduction; the use of dedicated synchrotron sources also allows a significant increase in data collection using smaller crystals, although obtaining the latter is still a major rate-limiting step in the process. These activities are expensive, however, and require large investments of capital or the formation of consortia of interested parties from the public or private sectors. In addition, the selection of targets for structural biology programs is currently biased towards those which are easy to purify and crystallize, at the expense of more challenging targets such as membrane proteins [despite recent success with rhodopsin (10)].

An alternative method of structure determination is nuclear magnetic resonance (NMR) in solution, which can capture the variety of conformations expected to be available for ligand interaction within purified proteins. Although technically more straightforward than X-ray crystallography, NMR has limitations, for example in the maximum size of protein that can be analyzed.

Given the large number of proteins for which structures are required, the only efficient way forward is to complement the structural proteomics initiatives with large-scale homology modeling. Programs such as ALIGN and MODELLER have 
been used successfully (11), and it is estimated that homology models covering half the human proteome may become available by 2003 (1).

\section{Protein Site Analysis}

The 3-D disposition of key binding atoms within the ligandbinding pocket of a protein is investigated by a process of site analysis. Ligands interact with their protein sites through a number of key molecular mechanisms, namely hydrogen bonding, electrostatics, hydrophobic, and Van der Waals interactions. Specificity of the ligand for the site is achieved by a complementary disposition of attractive forces between the molecular com ponents, while repulsion plays an important role in specificity by governing interactions at very short range. This is why replacement of a hydrogen atom by a methyl group can render a molecule inactive.

Since hydrogen-bonding interactions are strongly directional, hydrogen-bonding groups on the protein will provide key anchor points for complementary groups on the ligand. Hydrogen-bonding probability is geometrically disposed around a heteroatom and can be studied from surveys of crystal data (8). The probability data can be presented as maps on spherical shells projected onto the accessible surface of the site atoms. (The accessible surface is defined as the surface swept out by the centroid of a water molecule rolling over the Van der Waals surface of a site). In some instances, the maps produced from neighboring heteroatoms may overlap to produce a strongly hydrogen-bonding sub-region within the site.

On the other hand, hydrophobic interactions between a ligand and its site are not directional; however it is possible to allocate a hydrophobic score to grid points on the accessible surface of the site. Similarly, electrostatic potentials can be com puted on the accessible surface.

The analysis of sites can be automated to identify important site points such as the hydrogen-bonding atoms and hydrophobic sub-regions. These points are illustrated in Figures 2 and 3.

\section{Structure-Based Ligand Design}

The pharmaceutical industry faces a large increase in the number of therapeutic targets available for exploration. New methods must be developed for drug discovery that can be automated, are cheap, and optimally explore the vast chemical space available for drug design. It has been estimated that the number of drug-like molecular structures possible is $10^{60}(2)$. This number can be compared with the estimated mass of the visible universe at $10^{56} \mathrm{~g}$. In comparison, the number of different chemical structures synthesized is small and has not yet reached $10^{8}$. The reason for the huge numbers of potential molecular structures is the combinatorial way in which molecules can be assembled from their constituent atoms. Clearly, novel in silico methods will have to be developed to cope with reducing numbers of this magnitude to a few thousand compounds for screening.

\section{De Novo Design Processes}

After performing site analysis, the next step is to generate small molecule scaffolds within the binding site using algorithms for de novo design such as De Novo's Skelgen ${ }^{\mathrm{TM}}$. This is an automated structure generation algorithm that builds up molecular structures in the site from small fragments. The fragments can be taken from proprietary chemistries or from databases of compounds such as the World Drug Index. Fragment assembly rules ensure that the molecular structures are built with appropriate bonds and undesired structural arrangements are eliminated.

Molecular assembly is stochastic and therefore creates diverse structures (12). There are two general strategies for molecular assembly:

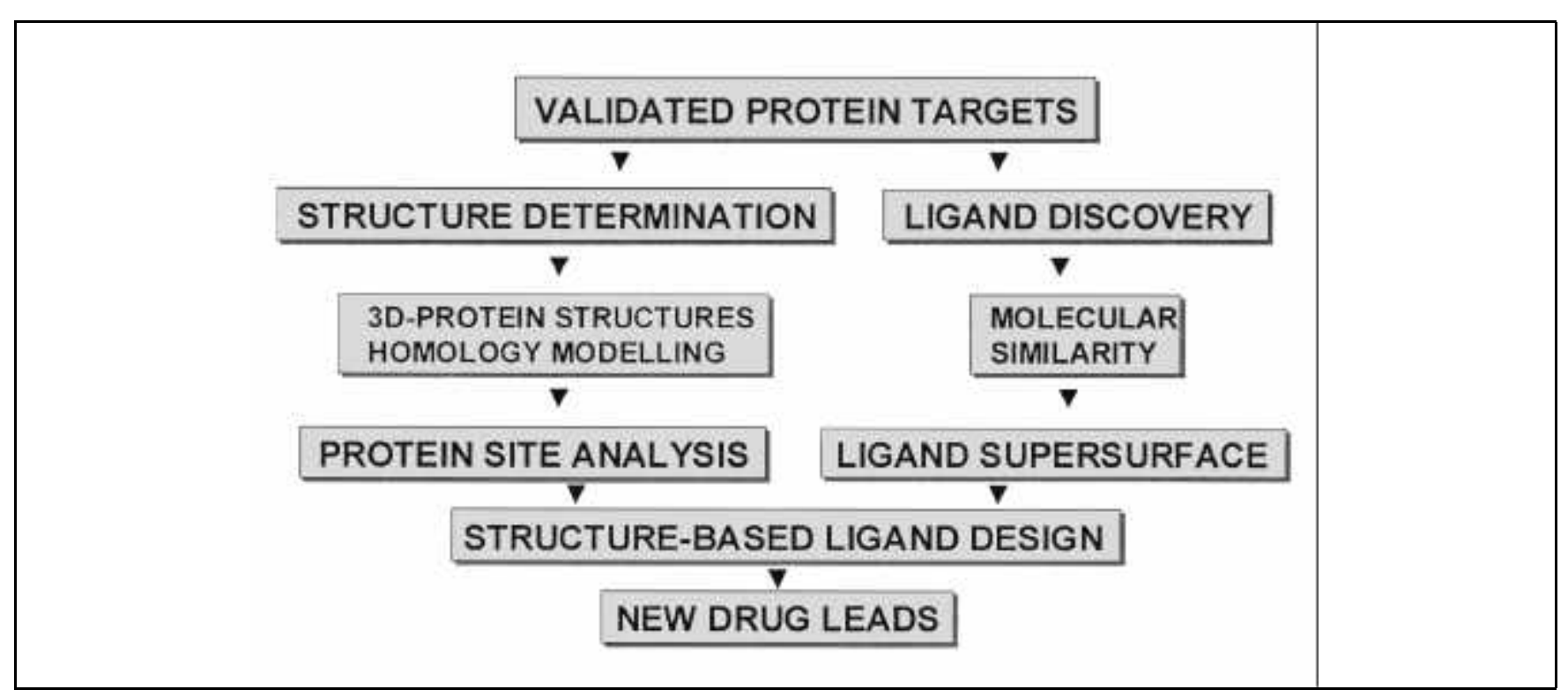

Figure 1. Flow of activity from protein target to small molecule. Virtual small molecule designs to ligand-binding sites on proteins are created from three-dimensional structural information (left hand side) or ligand information (right hand side). 
1. An outside-in strategy arranges fragments complementary to the local site point, initially placed at the edge of the site, and builds molecular structures within the remaining space to span between existing fragments. The whole process is a spanning strategy that tries to identify an inner core linking the fragments positioned at the exterior.

2. The opposite procedure, an inside-out strategy, places a randomly chosen fragment at a site point and assembles the structure away from that fragment. This is a random walk procedure evolving from the initial starting point.

Each step in the assembly process is scored for interaction with the site atoms. The evolution of a virtual molecular structure proceeds from the addition, removal, or replacement of a fragment; at each step the molecule is allowed to flex randomly and be moved by a random translation and orientational shift. Structure generation is a combinatoric process and thereby potentially giving rise to a vast output of virtual molecules from which to select optimally fitting structures. Skelgen ${ }^{\mathrm{TM}}$ uses simulated annealing for optimization by analogy with the annealing of glass.
Here, the system is heated and allowed to cool slowly so that the glass molecules are optimally aligned with each other. This method is capable of handling vast spaces, and has been used routinely at De Novo for optimizing problems with an excess of $10^{100}$ possible solutions.

The objective of simulated annealing is to find the global optimum, i.e., lowest energy state of molecules within the site. In principle, the method is ergodic, that is to say, the optimum can be reached irrespective of the starting position, since the trajectory is not trapped in local minima. This has been called a "hill climbing" algorithm where it is possible to escape from a local minimum by moving up an energy pathway and over a barrier to explore new regions. If the algorithm is stopped prematurely, however, a variety of near-global solutions are produced. This feature produces many different molecular structures to fit the site, thereby allowing a user to explore the molecular diversity of ligands within the ligand-binding pocket. Alternative optimization methods that could be used include genetic algorithms, evolutionary algorithms, and parallel tempering.

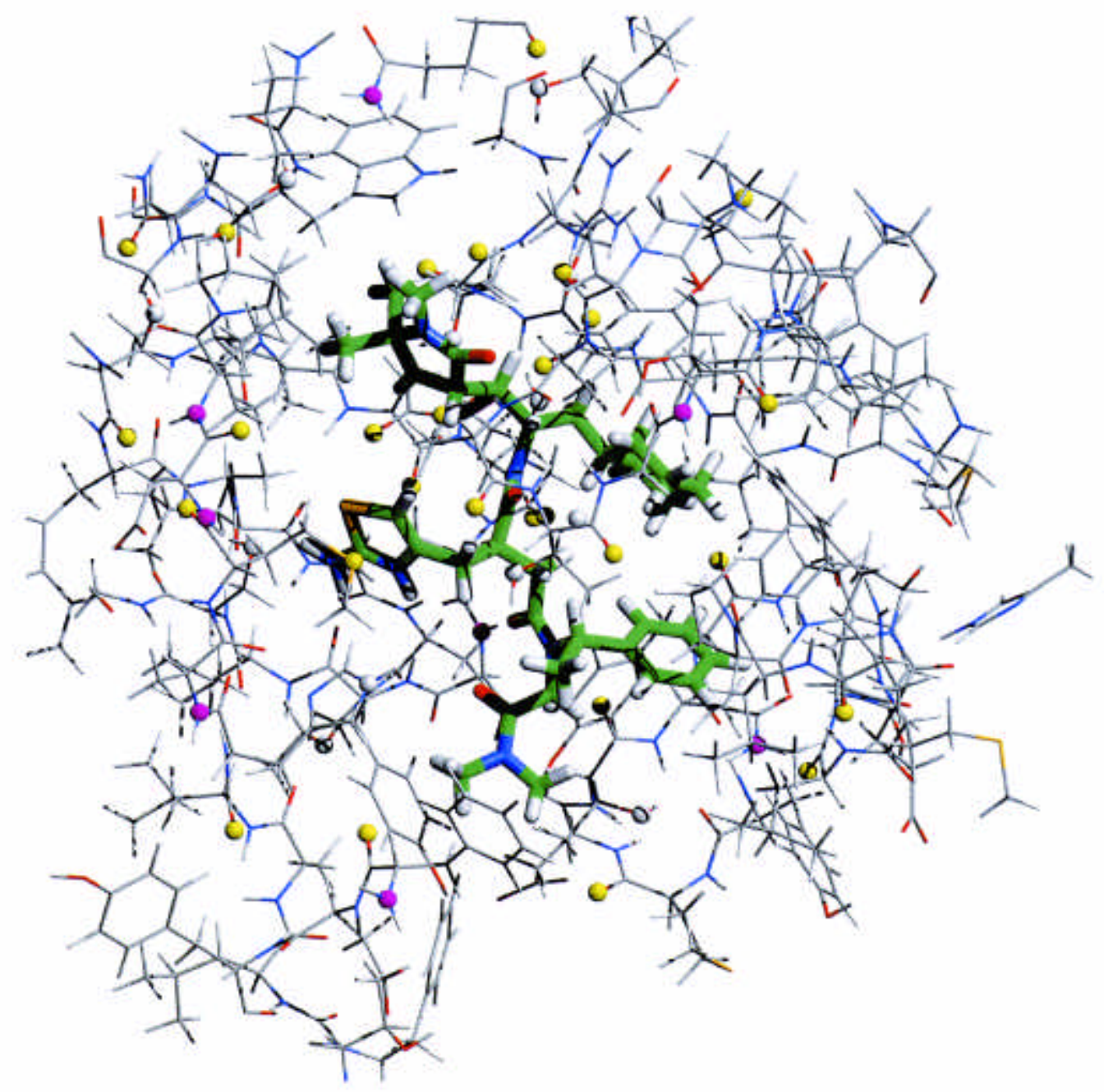

Figure 2. Hydrogen-bonding site points within the ligand-binding cavity of the aspartyl protease renin. A peptidic inhibitor is highlighted within the active site. Hydrogen-bonding site points are color-coded as mauve (donor), yellow (acceptor), and white (amphiprotic) spheres. 


\section{SCORING THE OUTPUT}

The optimizing techniques outlined above require a mathematical expression to describe the energy landscape. In structure generation, this is called the scoring function. The interaction energy between a drug molecule and its site can be determined experimentally from the equation

$$
\Delta G=-R T \ln K b
$$

where $\Delta G$ is the free energy of interaction, $K b$ is the experimental binding constant, $R$ is the gas constant, and $T$ is the temperature. The free energy has an enthalpic and entropic component defined by

$$
\Delta G=\Delta H-T \Delta S
$$

where $\Delta H$ is the change in enthalpy on binding and $\Delta S$ is the change in entropy. Entropic contributions to the free energy are hard to compute and can only be derived by large-scale simulations [e.g., Chau (3)]. The scoring function is determined by regression analysis using experimentally determined $\Delta G$ values together with crystal structural data. The weakness in the assessment of scoring functions is that the number of diverse ligand co-crystal complexes is small and that the range of $\Delta G$ values is limited. Since the function is not precise, there is much debate on how to improve it.

\section{FROM DE NOVO STRUCTURE TO COMBINATORIAL LIBRARY}

Automated structure generation procedures can be used to produce either unique structures that fit the site without the need for further computational effort, or partial scaffolds that can be used for further elaboration in silico. The scaffolds can then be assessed for suitability as chemical templates for focused libraries. Combinatorial chemistry can be harnessed to these in silico structure generation techniques, and the resulting virtual libraries built within the binding site to produce focused libraries. These could be used either for a particular site, or generally, for exploration of a class of sites such as a gene family. This process has the potential to open up the field of chemical genomics by design.

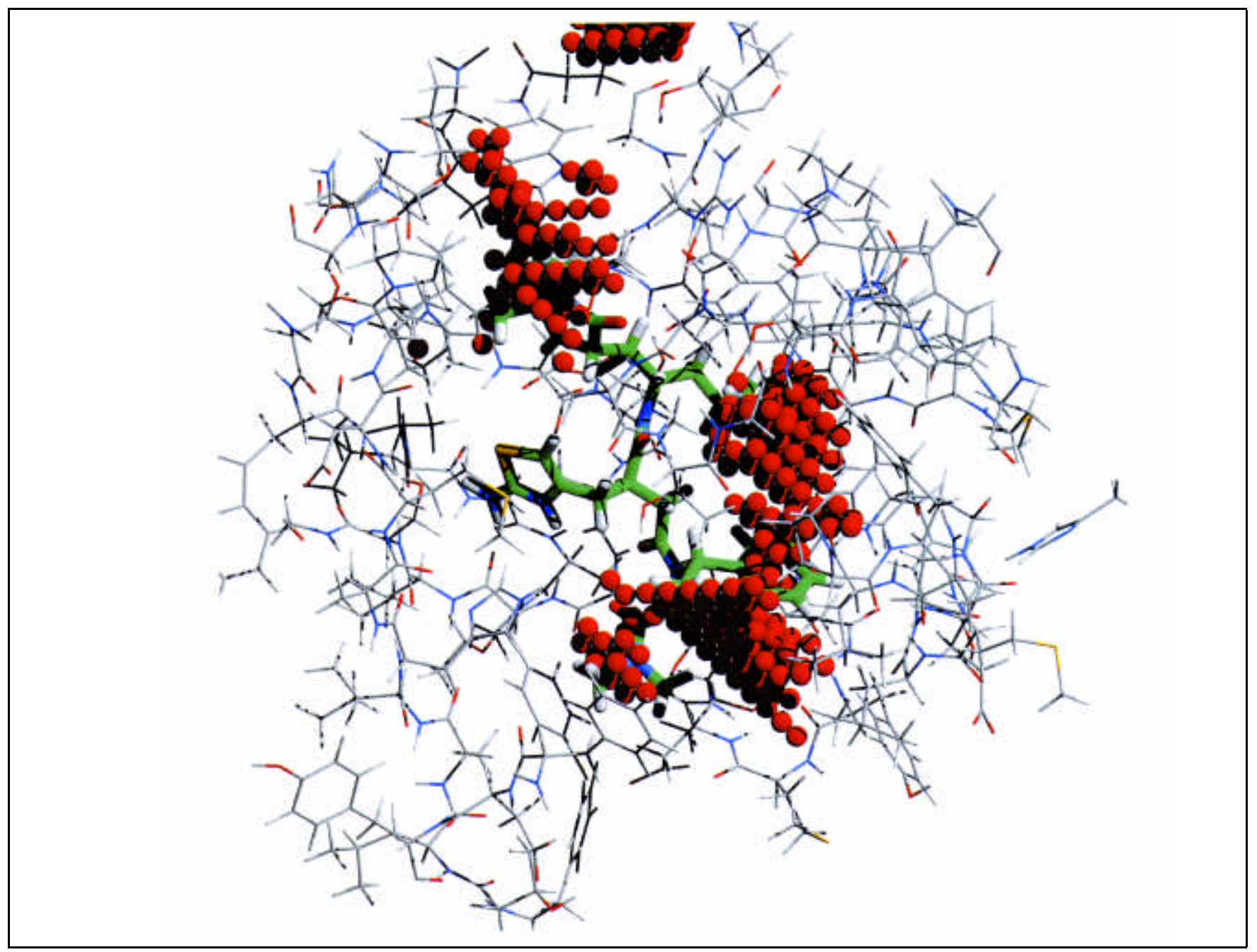

Figure 3. Hydrophobic site points within the ligand-binding cavity of the aspartyl protease renin. The same area is shown (with inhibitor) as for Figure 2. Red spheres represent hydrophobic site points. 
Figure 4 summarizes the de novo design process.

\section{LIGAND-BASED DESIGN}

The flow of activity from target to virtual small molecules, as described above, is dependent on having a 3-D protein structure on which to prime the design process. In many cases, this information is not available, for example with the important $G$ protein-coupled receptor (GPCR) family of drug targets. Fortunately, it is possible to create a virtual binding site using the site points identified by overlaying a series of known ligands to the protein, as described in the following sections.

\section{Ligand Discovery}

Screening by binding affinity or biological activity has been relatively successful in discovering small molecule ligands and forms the basis of most pharmaceutical discovery strategies. The drawback of this is the serendipitous nature of hit identification and the limited diversity of the compounds found in screening collections. The problems may be overcome by using de novo ligand-based design that utilizes existing compound data in the absence of a protein structure.

An algorithm, SLATE, has recently been described to identify molecular similarity for ligand-based design (9). The principle parts of the algorithm are summarized in Figure 5.

The aim of the algorithm is to infer the geometrical distribution of site points within the protein cavity from the corresponding disposition of ligand points, in effect creating a "virtual" cavity. The ligand points are automatically determined from a minimum number of compounds with known activity at the site. The next step in this process is to optimize the molecular similarity of these ligand points. Although this is a complex process, since only partial similarity will be apparent with the choice of ligands, a solution is outlined below.

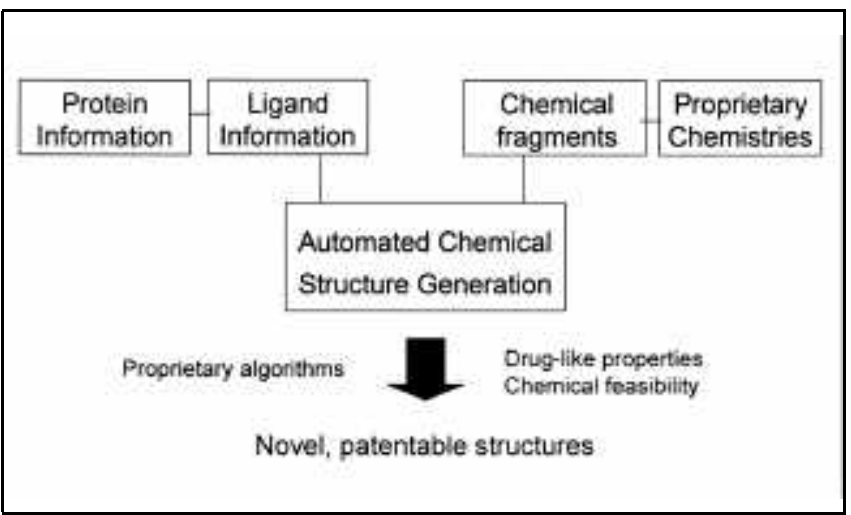

Figure 4. De novo structure generation. The input is three-dimensional data about the site for structure-based design or ligand information for ligand-based design. Small chemical fragments are also input to the algorithm. Structure generation proceeds by optimizing the positioning and linking of fragments with the appropriate chemistry within the site. The site constrains the objective function within the algorithm. Further constraints such as chemical feasibility and drug-like properties can be incorporated into the scoring function. The output is a set of novel and diverse structures.

\section{Molecular Partial Similarity}

Whole molecule comparisons cannot identify subtle similarities; what is needed is a method that identifies and maximizes partial similarity within a pair of structures. Certain features, such as hydrogen-bonding ligand points, hydrophobic regions, and the maxima and minima of electrostatic potential, need to be prioritized in the search for molecular similarity. In addition, the shapes of the interacting ligand surfaces need to be discriminated by the algorithm from those surfaces that face away into solution and therefore are not involved in the interactions.

To develop a similarity description, a matrix of all pairs of molecules in the set has to be derived. In addition, many ligands have torsion bonds that allow the molecules to be flexible, which has to be taken into account. At the end of the comparisons, the molecules in the set have to be superposed in an optimal way without preconception.

Molecular interaction features such as hydrogen bonds have to be added to the ligands at the start. Thus, if the molecule contains a carbonyl oxygen atom, the position of a donor heteroatom in the receptor will be at a distance of $2.9 \AA$ from the oxygen atom and along the direction of the carbonyl oxygen atom lone pairs. A table for the projection of features can be built and transferred to all ligand points in the molecules. These features become prioritized points for molecular similarity. Their positions are all represented as a distance matrix that

\section{Molecular definitions}

The following points for superposition are automa tically identified on the ligands:

- Ligand acceptor atoms
- Inferred receptor acceptor atoms
- Vectors normal to aromatic rings passing
through the centroids

\section{Objective function}

Sum of difference distance matrix elements for the critical points to:

- Allow null correspondences

- Allow flexibility around the torsion angles

- Optimize through simulated annealing

Output of SLATE is an optimally matched pair of molecules for partial molecular similarity

The algorithm is repeated for all molecular pairs

Figure 5. The essential features of the SLATE algorithm for superposing flexible ligands. 
changes as the molecules flex. These geometrical techniques can also be applied to aromatic rings.

Each pair of molecules is then assessed for the molecular partial similarity by computing the distance matrix for all the points in each molecule. Subsequently, a difference distance matrix is computed by subtraction of the two distance matrices. The partial match is identified from the optimal difference distance matrix using null correspondences.

\section{Coping with Flexibility}

A variety of random conformations are chosen as starting points and the difference distance matrix computed for each conformational comparison. During the matching process the conformations can flex; therefore the corresponding difference distance matrices are tracked and ranked to obtain the best matches. This optimization process uses simulated annealing. All pairs are analyzed and the results put into a similarity matrix to classify the matches for subsequent superposition.

Finally, molecular surfaces are matched using the algorithm PSEUDO. The matches are ranked for 50 different conformations of each molecule and optimal matches identified for each molecular pair.

\section{Creation of a Ligand Supersurface}

From the partially similar molecular surfaces, the molecular supersurface is computed using McLachlan's MATFIT algorithm (7). This supersurface is then used as the constraining surface for novel ligand design.

The output of the SLATE algorithm has been been compared with the orientation of ligands found in crystal structures. For example, the superpositions of 6 thermolysin ligands are close to those found in the crystal with 8 of the interacting atoms lying within $1 \AA$ (9). The method has been used successfully to construct a pharmacophore model for the histamine $\mathrm{H} 3$ receptor and to design high affinity antagonists (5).

\section{CONCLUSION}

Proteomics will provide a large number of validated targets for drug design, but optimal methods have to be created to handle this challenge. Where possible, in silico solutions should be employed before the committal of expensive chemical resources. Automated structure-based design and its ligand-based cousin need to be honed for routine application to any target. With small binding sites, such as those found at enzyme active sites or small binding sites on receptors, the process of in silico design is particularly useful. These techniques provide an essential toolkit for transforming proteomics data into pharmaceutically relevant small molecules. As well as this obvious utility for pharmaceutical discovery, the wider application of small molecule probes for the entire proteome will accelerate our understanding of protein structure and function at a genomic level.

\section{REFERENCES}

1.Baker, D. and A. Sali. 2001 Protein structure prediction and structural ge- nomics. Science 294:93-96.

2.Bohacek, R.S., C. McMartin, and W.C. Guida. 1996. The art and practice of structure-based drug design: a molecular modelling perspective. Med. Res. Rev.16:3-50.

3.Chau, P-L. 2001. Process and thermodynamics of ligand-receptor interaction studied using a novel simulation method. Chem. Phys. Lett. 334:343-351.

4.Dean, P.M., E.D. Zanders, and D.S. Bailey. 2001. Industrial-scale, genomics-based drug design and discovery. Trends Biotechnol. 19:288-292.

5.De Esch, I.J., J.E. Mills, T.D. Perkins, G. Romeo, M. Hoffmann, K. Wieland, R. Leurs, W.M. Menge, P.H. Nederkoom, P.M. Dean, and H. Timmerman. 2001. Development of a pharmacophore model for histamine $\mathrm{H} 3$ receptor antagonists, using the newly developed molecular modelling program SLATE. J. Med. Chem. 44:1666-1674.

6.International Human Genome Sequencing Consortium. 2001. Initial sequencing and analysis of the human genome. Nature 409:862-921.

7.McLachlan, A.D. 1979. Gene duplications in the structural evolution of chymotrypsin. J. Mol. Biol. 128:49-79.

8.Mills, J.E., T.D. Perkins, and P.M. Dean. 1997. An automated method for predicting the positions of hydrogen-bonding atoms in binding sites. J. Comput. Aided Mol. Des. 11:229-242.

9.Mills, J.E.J., I.J.P. De Esch, T.D.J. Perkins, and P.M. Dean. 2001. SLATE: a method for the superposition of flexible ligands. J. Comput. Aided Mol. Des. 15:81-96.

10.Palczewski, K., T. Kumasaka, T. Hori, C.A. Behnke, H. Motoshima, B.A. Fox, I. Le Trong, D.C. Teller, et al. 2000. Crystal structure of rhodopsin: a G protein-coupled receptor. Science 289:739-745.

11.Sali, A. and T.L. Blundell. 1993. Comparative protein modelling by satisfaction of spatial restraints. J. Mol. Biol. 234:779-815.

12. Todorov, N.P. and P.M. Dean. 1997. Evaluation of a method for controlling molecular scaffold diversity in de novo ligand design. J. Comput. Aided Mol. Des. 11:175-192.

13.Venter, J.C., M.D. Adams, E.W. Myers, P.W. Li, R.J. Mural, G.G. Sutton, H.O. Smith, M. Yandell, C.A. Evans, R.A. Holt, et al. 2001. The sequence of the human genome. Science 291:1304-1351.

Address all correspondence to:

Edward D. Zanders

De Novo Pharmaceuticals Ltd.

St. Andrews House

59 St. Andrews Street

Cambridge CB2 3DD, UK

e-mail:Ed.Zanders@denovopharma.com 